\title{
A REDUCIBILITY PROBLEM FOR THE CLASSICAL RESIDUE FORMULA
}

\author{
YU. I. LYUBICH \\ Department of Mathematics, Technion, 32000 Haifa, Israel \\ E-mail:lyubich@tx.technion.ac.il
}

Let $z_{1}, \ldots, z_{n}$ be $n$ distinct points in $\mathbb{C}$ and let

$$
G(z)=\prod_{k=1}^{n}\left(z-z_{k}\right)
$$

Denote by $\Gamma$ a simple contour surrounding $\left\{z_{k}\right\}_{k=1}^{n}$. The residue formula

$$
\frac{1}{2 \pi i} \int_{\Gamma} f(z) \frac{G^{\prime}(z)}{G(z)} d z=\sum_{k=1}^{n} f\left(z_{k}\right)
$$

is valid in a class of analytic functions, in particular, it is true for all polynomials of degree $\leq 2 n-1$. In this sense (1) is a Gauss type quadratic formula of order $n$.

Definition 1. Let $m$ be an integer, $2 \leq m \leq n$. A configuration $\left\{z_{k}\right\}_{k=1}^{n}$ is called $m$-reducible if there exists another configuration $\left\{w_{j}\right\}_{j=1}^{m}$ such that

$$
\frac{1}{n} \sum_{k=1}^{n} f\left(z_{k}\right)=\sum_{j=1}^{m} \alpha_{j} f\left(w_{j}\right), \quad f \in \mathcal{P} \text { ol }(\mathbb{C}), \quad \operatorname{deg} f \leq 2 m-1,
$$

with some complex coefficients $\alpha_{1}, \ldots, \alpha_{m}$. Obviously, (2) implies that $\sum_{j=1}^{m} \alpha_{j}=1$.

REMARK. It does not make sense to extend Definition 1 to $m=1$ since in this case the barycenter $w_{1}$ of the system $\left\{z_{k}\right\}$ satisfies (2) with $\alpha_{1}=1$. Thus one can say that every configuration is 1-reducible.

Definition 2. A configuration $\left\{z_{k}\right\}_{k=1}^{n}$ is called irreducible if for each $m \in[2, n)$ it is not $m$-reducible.

Note that these properties are affine invariant, i.e. they are invariant with respect to transformations $z \mapsto a z+b$.

It is shown in [1] that a triangle $\left\{z_{k}\right\}_{k=1}^{3}$ is irreducible if and only if it is either equilateral or isosceles with the angle between the equal sides which is equal to

$$
\alpha=\frac{\pi}{2}+\arctan \frac{\eta}{\sqrt{4-\eta^{2}}}
$$


where $\eta$ is the unique real root of the cubic equation

$$
4 \eta^{3}-12 \eta^{2}+9 \eta+2=0
$$

(so that $\eta \approx 0,5283 \pi$ ).

Also it turns out that for every $n \in \mathbb{N}, n \geq 3$ the regular $n$-gon is irreducible. It would be interesting to find other examples for $n \geq 4$ and, maybe, to describe explicitly all of them for small $n$. In general there is a characterization of irreducibility by a union of systems of algebraic equations. (This can be easily extracted from [1, Theorem 6].)

CONJECTURE. For every $n$ the set of irreducible configurations is finite up to affine equivalence.

To support formally this conjecture let me indicate that each system mentioned above consists of $n-2$ equations. On the other hand, the affine class of $n$-configuration depends exactly on $n-2$ complex parameters.

\section{References}

[1] Yu. I. Lyubich, Gauss type quadrature formula, power moment problem and elliptic curves, Mat. Fizika, Analiz, Geometriya 9 (2002), 128-145. 